\title{
Journal of Automation and Control Engineering
}

\section{CONTENTS}

\section{Volume 1, Number 3, September 2013}

Application of Street Tracking Algorithm to Improve Performance of a Low-Cost INS/GPS System 181

Thang Nguyen Van, Trinh Chu Duc, and Tan Tran Duc

Performance Improvement Technique for Induction Motor Driven by a Matrix Converter under Abnormal Input Conditions 186

Nguyen Khanh Tu Tam, Nguyen Van Nho, Huynh Thai Hoang, and Vu Duy Nhat

Spatio-Temporal Web Sensors Using Web Queries vs. Documents

Shun Hattori

ATTORNEY 209: A Virtual Assistant Adviser for Family-Based Cases 198

Kristine Sarah B. Agreda, Eliz Danielle B. Fabito, Lyzel C. Prado, Ma. Hannah G. Tebelin, and Benilda

\section{Eleonor V. Comendador}

Recommendation System Keeping Both Precision and Recall Based on Uninteresting Information. 202

Tsukasa Kondo, Fumiko Harada, and Hiromitsu Shimakawa

Extracting Student Motivation Factors in Education with Contextual Inquiry .208

Wataru Takahashi, Fumiko Harada, Hiromitsu Shimakawa, and Takahiro Koyama

Face Clustering Experiments on News Video Images

Subhradeep Kayal

Two-Level Cooperation in Autonomic Cloud Resource Management

Giang Son Tran, Laurent Broto, Daniel Hagimont, and Alain Tchan

Speech Recognition in Human-Computer Interactive Control.

Vu Duc Lung, Phan Dinh Duy, Nguyen Vo An Phu, Nguyen Hoang Long, and Truong Nguyen Vu

Study on Mechanical Adaptive Design, Construction and Control of Knee Continuous Passive Motion Machine

Ngoc-Bich Le, Huu-Nghia Nguyen, Duy-Anh Nguyen, and Hoang-Duy Vo

A Multisensor Approach for Error Compensation in CNC Machine Tools

Abderrazak El Ouafi and Noureddine Barka

Design for the Predictor of the Emergency Braking System Based on Fuzzy Algorithm

J. H. Li and H. M. Kim

Hardware Design and Simulation of Reduced-Order Extended Kalman Filter Estimator and Speed Fuzzy Controller for Sensorless PMSM Drives

Nguyen Vu Quynh, Ying-Shieh Kung, Chung-Chun Huang, and Liang-Chiao Huang

High Throughput- Resource Saving Hardware Implementation of AES-CCM for Robust Security Network .250

Dang Khoa Nguyen, Leonardo Lanante, and Hiroshi Ochi 
On the Development of a Specialized Flexible Gripper for Garment Handling. .255

Thuy-Hong-Loan Le, Michal Jilich, Alberto Landini, Matteo Zoppi, Dimiter Zlatanov, and Rezia Molfino

Comparative Study of Forces Involved in Different Styles of Handwriting .260

Gnanasambandam Kulothungan, Meyyappan Nachiappan, Mukund Suresh Kumar, and Sharmilaa Rajasekaran

Robustness of Adaptive Fuzzy for PMSM Sensorless Speed Controller .265 Nguyen Vu Quynh, Ying-Shieh Kung, and Lam Thanh Hien

Discovery of Memorized Features from Web Browsing History for Re-finding Vaguely-Memorized Pages ..270 Shinichi Ikura, Tsukasa Kondo, Fumiko Harada, and Hiromitsu Shimakawa

Speech Recognition on Robot Controller Implemented on FPGA .274

Phan Dinh Duy, Vu Duc Lung, Nguyen Quang Duy Trang, and Nguyen Cong Toan 